\title{
Performance of MIMO Systems Using Space Time Block Codes (STBC)
}

\author{
Christopher M. Lau \\ Fairleigh Dickinson University, Teaneck, NJ, USA \\ Email: chris_lau10@outlook.com
}

How to cite this paper: Lau, C.M. (2021) Performance of MIMO Systems Using Space Time Block Codes (STBC). Open Journal of Applied Sciences, 11, 273-286.

https://doi.org/10.4236/ojapps.2021.113020

Received: February 6, 2021

Accepted: March 13, 2021

Published: March 16, 2021

Copyright $\odot 2021$ by author(s) and Scientific Research Publishing Inc. This work is licensed under the Creative Commons Attribution International License (CC BY 4.0).

http://creativecommons.org/licenses/by/4.0/

\begin{abstract}
Digital Communications, in relation to wireless networks, have taken off in recent years due to the expanding need to communicate faster and more efficiently. A popular way to achieve this is by using wireless Multiple Input Multiple Output (MIMO) communication systems. MIMO systems utilize Space Time Block Codes (STBC) as one of the leading ways to obtain higher data rates with limited bandwidth and power. With several STBC methods currently available, this paper analyzes simulations using Orthogonal Space Time Block Codes (OSTBC) in Rayleigh fading channels to evaluate the performance of MIMO systems. The selection to use a Rayleigh fading channel as a model for a non-line-of-sight (nLOS) environment is selected to mimic installations where a large number of signal paths and reflections are expected. All simulations are coded, generated and plotted using MATLAB resulting in graphical data representing the bit-error rate (BER) to signal-to-noise ratio $\left(\mathrm{E}_{\mathrm{b}} / \mathrm{N}_{0}\right)$ or SNR. Each simulation captures how different configurations of key variables including code rate, diversity and antenna count can impact system performance. Four modulation schemes (BPSK, QPSK, 16-QAM and 64-QAM) are included in each simulation. Conclusive evidence based upon these simulations suggests higher diversity gains were achieved with a greater number of antennas. The most significant factor for increasing system performance was using a lower count of transmit antennas with a higher count of receive antennas.
\end{abstract}

\section{Keywords}

Computer Analysis, Digital Communications, MATLAB, MIMO, Modulation Techniques, Multiple Input Multiple Output Systems, Orthogonal Space Time Block Codes, OSTBC, Rayleigh Fading Channels, Space Time Block Codes, STBC, Wireless Communications, Wireless Networks 


\section{Introduction}

Multiple input multiple output (MIMO) systems are an efficient way to transmit large amounts of reliable data to multiple users in a wireless system. In contrast to a single input single output system (SISO), MIMO systems utilize multiple antennas. MIMO systems have caught the eyes of many researchers and scientists as it outperforms SISO systems in many ways [1]. Markets invested in 5G cellular and next-generation devices [2] will be reliant upon MIMO systems for the increased bandwidth capabilities in order to serve as many subscribers or end users as possible. MIMO systems can achieve increased bandwidth by enabling spatial multiplexing and diversity. Tremendous amounts of work have been investigated and documented over the last decade exploring MIMO transmission techniques such as beam steering, spatial channels, and space-time coding.

Space Time Block Codes (STBC) is a form of diversity coding that are capable of distinguishing transmissions over multiple paths. Some well-known STBCs are Alamouti and orthogonal schemes which deal with multiple transmit and receive antennas. STBC is designed to allow for increased performance of wireless transmissions knowing each signal may be delayed or experience different signal-to-noise ratios (SNR). STBC compensates for added noise and other additional losses through spatial channels and can help reduce bit-error rates. An important way to study and analyze such performance is to visually inspect graphical data. Simulations performed with MATLAB in Section 5 will explore the use of Orthogonal Space Time Block Codes (OSTBC) in Rayleigh fading channels to help evaluate the performance of MIMO systems. Even though theoretical and real-world results may vary, conclusive findings will be examined throughout this paper.

The increased number of antennas in a MIMO system unlike its single antenna system counterpart, can use multiple channels for increased redundancy providing each channel with independent delay and losses. A common way to analyze performance based upon the number of bits is to calculate the bit-error rates (BER) using a variety of modulation techniques including BPSK, QPSK, 16-QAM and 64-QAM.

It is important to realize that MIMO systems will help achieve a higher capacity for data rates and much more. An advantage to these systems is the demand for higher bandwidth is not needed as the entire system runs on the same frequency. A common misconception for power requirements is if multiple transmit and receive antennas are used, then it would be consuming more. The total power consumption does not exceed the power of any SISO system. As the world evolves, the use of MIMO systems is being implemented globally and deployed at very large scales. MIMO techniques promote and enable non-line-of-sight installations, reduced electrical demands, lower BER, increased network range and lower levels of interference. Base and mobile stations from cellular carriers use a low number of antennas to drive a simpler, more cost-efficient design. 


\section{Basic Design Concepts}

Receiver diversity is used in present cellular mobile systems to gain benefits for improving quality and uplink range. Error control coding can be combined with transmit diversity to achieve improved performance of multiple antenna transmission systems leading to coding gain advantages and diversity benefits. A drawback to this method is bandwidth expansion due to increased levels of code redundancy. Compiling error control coding with modulation and transmit diversity as a single block needs to be performed with space time codes. Space time coding makes it possible to achieve coding gain as well as diversity benefits without bandwidth expansion [3]. Some basic fundamental concepts of MIMO systems begin with understanding how they are used in the field.

With multiple antennas and receivers come multiple scenarios for transmission paths to propagate along. This multipath effect benefits the subscriber or end user. MIMO systems can be broadly classified into three main categories: Space Diversity, Spatial Multiplexing, and Beamforming techniques. Beamforming is when the transmitter and receiver can aim their main beam at each other. This will increase the received power consequentially increasing the SNR. In order to reduce the probability of an outage, spatial diversity can be used to code a signal through the transmit antennas to create redundancy. Spatial multiplexing allows transmission in parallel with use of all the transmit antennas. The receiver will then choose which signal to process accordingly. STBC is a very powerful encoding scheme to fill the gap between spatial multiplexing and space time diversity [4].

For the case where an antenna array cannot be used with the receivers, one should consider using an antenna array with the transmitters. This provides transmit diversity for the wireless system. Although receiving diversity scheme has a $3 \mathrm{~dB}$ bit-error rate (BER) performance gain when compared with a transmit diversity scheme [5], transmit diversity is very attractive for downlink wireless systems, because using antenna array is usually not possible for the mobile unit [6].

\subsection{System Design}

The path antennas take to communicate with one another will be denoted as a channel. We begin with matrix $H$, Equation (1), which can be represented as a complex matrix of the wireless channels between the receive and transmit antennas. All transmit antennas will be denoted as $T_{n}$ and receive antennas as $N_{r}$ creating a matrix $H=N_{t} \times N_{r}$ STBC are generally put into a matrix where $H$ denotes the channel matrix of a STBC with ${ }^{\star}$ representing the conjugate with the paths of each channel making up the following equation:

$$
\boldsymbol{H}=\left(\begin{array}{cccc}
h_{1} & h_{2} & h_{3} & h_{4} \\
-h_{2}^{*} & h_{1}^{*} & -h_{4}^{*} & h_{3} \\
-h_{3}^{*} & -h_{4}^{*} & h_{1}^{*} & h_{2}^{*} \\
h_{4} & -h_{3} & -h_{2} & h_{1}
\end{array}\right)
$$


The channels can be shown as $h_{\mathrm{i}, \mathrm{j}}$ with $i$ being the $i^{\text {th }}$ transmitter and $j$ being the $f^{\text {th }}$ receiver as shown in Figure 1. The signal model for MIMO system identified above is shown in Equation (6) where vectors $y, s$ and $\boldsymbol{n}$ being received, transmitted and noise vectors, respectively. Noise can be a number of different things, but we will assume white Gaussian noise for this paper. Noise is added to each channel as the receiver picks up the transmitted signal. The receiver can do this by taking the transmit signals and putting them into a set of linear equations to which it can solve for. The only factor preventing this from working is when correlation between signals begin. Prevention of correlation can be accomplished by utilizing a proper number of transmitters and receivers as well as considering the installation spacing between each antenna.

Generally, it is good practice to take the carrier frequency and divide this by 2 which will result in the minimum spacing between each component to be mathematically represented as $f_{d} / 2$. The channel matrix in a MIMO system has a significant impact on performance. The code rate of a STBC, with the code word matrix $X$, is defined as $R=k / T$ symbols per channel use where k is the number of independent information symbols embedded in $\boldsymbol{X}$ [7]. An STBC is said to be full-rate or high-rate if $R=1$ or $R>1$, respectively.

\subsection{Channel Design}

Approaching channel design can be very tricky especially for the intended use of the system. Two major techniques are as follows: designing a channel matrix in relation to the environment or by taking a more analytical approach. The channel matrix physical model would depend on physical parameters such as the angle of arrival (AOA), angle of departure (AOD), and time of arrival (TOA). The environment can influence design in several ways including transmit/receive angles, time taken to travel from transmitter to receiver, etc. The more independent channel approach treats each case as if they were in isolation of one another. The lack of dependency can easily create unwanted behavior and presents issues in real-world environments. The problem that arises is the creation of spatial correlation amongst the transmitter and receiver.

\subsection{Relevance}

The total transmit power of a MIMO system is dispersed among all the transmitter antennas in the system. This allows for a gain that improves spectral

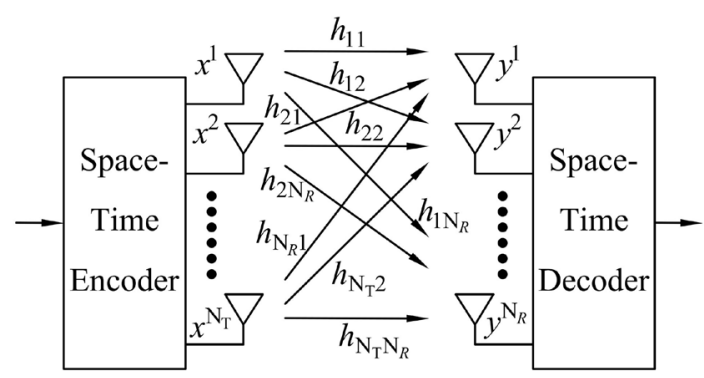

Figure 1. Typical MIMO system design. 
efficiency which can increase the bit per second per Hertz of bandwidth. It is important to note that each antenna element in a MIMO system operates on the same frequency and therefore does not require extra bandwidth. Channel design greatly depends on the surroundings in terms of the environment. Transmitters and receivers are set up into two main configurations: Line-of-sight (LOS) and non-line-of-sight (nLOS).

With population growth and buildings expanding upward, crowded environments are slowly being converted to nLOS systems because it is impossible for antennas to be positioned this way. One can imagine LOS as it sounds with one antenna seeing the other antenna without any obstacles in between. All simulations that were performed use Rayleigh fading channels for the assumption of a rich scattering environment. This will improve the probability of multipath fading making nLOS the best choice since the received signals follow a Rayleigh distribution. Common cellular installations are representative of nLOS wireless radio propagation and hence the MIMO channel capacity has been investigated for Rayleigh fading channel models [3].

\section{Space Time Block Coding}

Channel fading is a problem in MIMO systems but there are ways to address this issue. What happens when you ask someone a question and they did not hear it? The person asking the question will repeat the original question. This simple concept can be applied to the transmitter and receiver antennas used by sending the data information over and over in hope that all the data is transmitted and received without any errors. Fading can be mitigated by diversity, which means the information is transmitted not only once but several times, hoping that at least one of the replicas will not undergo severe fading. The loss of information is decreased a great deal using this method. The diversity order or diversity gain of a MIMO system is defined as the number of independent receptions of the same signal. A MIMO system with $N_{t}$ transmit antennas and $N_{r}$ receive antennas has potential to achieve full diversity (i.e. maximum diversity) gain equal to $N_{t} \times$ $N_{r}[8]$.

We can call the transmission of information over and over as replicas which are generated by an encoder. The different replicas sent for exploiting diversity are generated by a space-time encoder which encodes a single stream through space using all the transmit antennas and through time by sending each symbol at different times [8]. This is the foundation of space-time coding which sole purpose is to achieve high reliability of data transmissions. Each STBC code is introduced by its corresponding generation matrix as illustrated in Figure 2.

This matrix maps the input symbols into space and time i.e. it determines the transmitting symbols from different antennas in each time slot. Most properties of the code such as diversity, code gain, rate, complexity of the detector, number of transmit antennas and time delay are given by this matrix. As an example, Equation (4) demonstrates the generation matrix of one of a complex orthogonal 


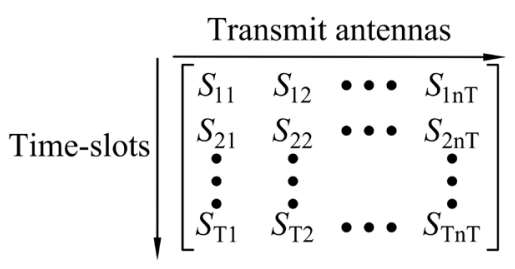

Figure 2. Transmit antenna and time slot relation to signal matrix in MIMO system with STBC.

STBC called the Alamouti code. The challenge of transmit diversity for multiple fading channels has been solved with several novel signal processing and error correction coding techniques. Prominent among these are space-time coding methodologies, including space-time trellis coding (STTC) [9], and STBC, which integrate the techniques of antenna array spatial diversity and channel coding.

\subsection{Alamouti's STBC}

STTC performs well in slowly fading environments but decoding complexity increases exponentially with the number of antennas. The alternative multi-antenna transmit diversity concept of STBC emerged in the work of Alamouti [6]. Alamouti code is the first scheme to be used in STBC systems presenting itself with an easy structure and simple transmit diversity. A man by the name of Siavash M. Alamouti was said to have been the first publication of STBCs ever. His first publication was in 1998 to which paved the way for intense research on the subject. In [2], he talks about a simple transmit diversity technique that has grown into hundreds of publications based off this single concept. The Alamouti scheme consists of just two transmit antennas with $N_{r}$ receive antennas to achieve a maximum order of diversity equal to $2 N_{r}$ [10]. Historically, the Alamouti code is the first STBC that provided full diversity at full data rate for two transmit antennas [1]. The information bits are first modulated using a digital modulation scheme. Then the encoder takes the block of two modulated symbols $\left(s_{1}\right.$ and $\left.s_{2}\right)$ in each encoding operation and hands it to the transmit antennas according to the code matrix.

This scheme uses two transmit antennas and one (or more) receive antennas. It has the same diversity order of 2 as the traditional maximal ratio receiver combining (MRRC) scheme with one transmit antenna and two receive antennas. Since there are only two transmit antennas, it can ideally achieve a code rate of 1 since it can transmit 2 symbols every 2 intervals. This is made possible by the orthogonal design of the system, and with just 2 transmit antennas, the encoder and decoder generally do not need to be a complex system. With the number of receiver antennas being infinite, we will assume the case when two receive antennas are used as shown in Figure 3. Matrix $G_{x}$, where $x$ represents the number of rows or coding schemes, is generally used to represent the encoding process. 


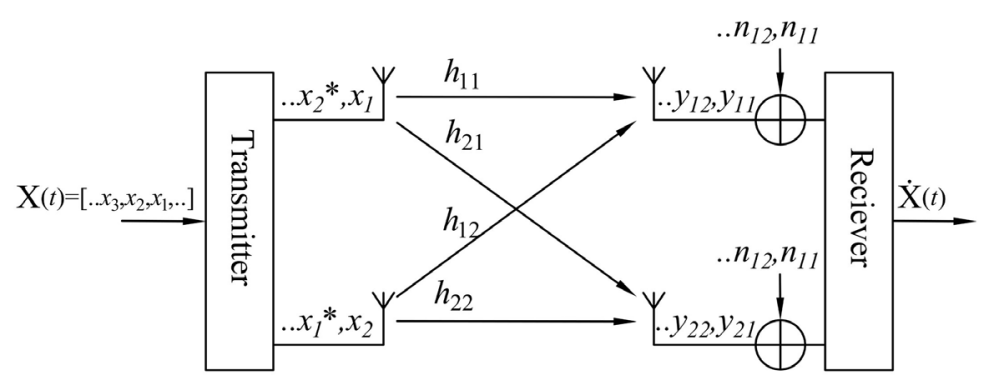

Figure 3. An Alamouti scheme of a $2 \times 2$ MIMO system with STBC.

This matrix $\boldsymbol{G}_{2}$ consists of two rows, each representing a different time slot, and two columns that represent each antenna transmitted symbol. Each symbol can be shown as $s_{1}$ and $s_{2}$ respectively. At a time $(t)$, symbols $s_{1}$ and $s_{2}$ are transmitted from antenna 1 and antenna 2. Assuming that each symbol has duration (T), then at time $(t+T)$, symbols $-s_{2}^{*}$ and $s_{1}^{*}$, where $\left(^{*}\right)$ denotes the complex conjugate, are transmitted from antenna 1 and antenna 2 respectively [2].

$$
\boldsymbol{G}_{2}=\left(\begin{array}{cc}
s_{1} & s_{2} \\
-s_{2}^{*} & s_{1}^{*}
\end{array}\right)
$$

The first row in Equation (2) represents the first transmission period and the second row represents the second transmission period. During the first transmission, symbols $s_{1}$ and $s_{2}$ are transmitted simultaneously from antenna 1 and antenna 2. In the second transmission period, symbol $-s_{2}^{*}$ is transmitted from antenna 1 and symbol $s_{1}^{*}$ from antenna 2 . It is clear that the encoding is performed in both time (two transmission intervals) and space domain (across two transmit antennas). Using two receive antennas will result in two independent receive signals that can be denoted as follows:

$$
\begin{aligned}
& r_{1}\left(t_{1}\right)=h_{1,1} s_{1}+h_{1,2} s_{2}+n_{1}\left(t_{1}\right) \\
& r_{1}\left(t_{2}\right)=-h_{1,1} s_{2}^{*}+h_{1,2} s_{1}^{*}+n_{1}\left(t_{2}\right) \\
& r_{2}\left(t_{1}\right)=h_{2,1} s_{1}^{*}+h_{2,2} s_{2}^{*}+n_{2}\left(t_{1}\right) \\
& r_{2}\left(t_{2}\right)=-h_{2,1} s_{2}^{*}+h_{2,2} s_{1}^{*}+n_{2}\left(t_{2}\right)
\end{aligned}
$$

These combine into:

$$
\begin{aligned}
& s_{1}=h_{1,1}^{*} r_{1}\left(t_{1}\right)+h_{1,2} r_{1}^{*}\left(t_{2}\right)+h_{2,1}^{*} r_{2}\left(t_{1}\right)+h_{2,2} r_{2}^{*}\left(t_{2}\right) \\
& s_{2}=h_{1,2}^{*} r_{1}\left(t_{1}\right)+h_{1,2} r_{1}^{*}\left(t_{2}\right)+h_{2,2}^{*} r_{2}\left(t_{1}\right)+h_{2,1} r_{2}^{*}\left(t_{2}\right)
\end{aligned}
$$

This can be finalized with substituting into:

$$
\begin{aligned}
& s_{1}=\left(\alpha_{1,1}+\alpha_{1,2}+\alpha_{2,1}+\alpha_{2,2}\right) s_{1}+h_{1,1}^{*} n_{1}+h_{1,2} n_{1}+h_{2,1}^{*} n_{2}+h_{2,2} n_{2}^{*} \\
& s_{2}=\left(\alpha_{1,1}+\alpha_{1,2}+\alpha_{2,1}+\alpha_{2,2}\right) s_{2}-h_{1,1} n_{1}+h_{1,2}^{*} n_{1}-h_{2,1} n_{2}^{*}+h_{2,2}^{*} n_{2}
\end{aligned}
$$

The final values of the transmit signals $s_{1}$ and $s_{2}$ are sent to a Maximum Likelihood (ML) decoder where the symbols can be estimated. The use of a single antenna can be utilized while still presenting a full diversity of 2 . With diversity being an optimal value, channel fading does not cause any significant issues. This is advantageous for using only one antenna at a mobile station. In cases 
where two transmit antennas and $N_{r}$ receivers are used, full diversity can also be achieved. What happens if there are more than two transmit antennas? Many unwanted variables such as interference, whether it be from other users or other paths within the system, will arise. Diversity plays a significant role in a system's performance. Also, Alamouti STBC can be applied to noncoherent FSK modulation too. In [10], some existing noncoherent detection methods for STBC are reviewed and new techniques are explained.

\subsection{Orthogonal Space Time Block Codes (OSTBC)}

With full diversity being ideal, other techniques known as OSTBC are used. It has been determined that full diversity is only achieved with real orthogonal codes. The essential feature of STBC is its inherent orthogonality, thus guaranteeing that a simple linear decoding technique generates the ML result. Complex orthogonal codes do exist, but full diversity is not beneficial with any more than two transmit antennas. With three antennas, block coding using this scheme can have $1 / 2$ or $3 / 4$ code rate and full diversity of $3 N_{r}$ The code with $1 / 2$ rate would allow 4 symbols to transmit every 8-time slots.

Equation (6) can be applied to any system model of a MIMO system where:

$$
\boldsymbol{y}=\boldsymbol{H} \boldsymbol{s}+\boldsymbol{n}
$$

With each entry representing a transmitted signal, $y=$ received signal vector, $\boldsymbol{H}=$ Channel matrix, $\boldsymbol{s}=$ column vector, and $\boldsymbol{n}=$ Gaussian white noise. The received signals are denoted by $R$ which is a $N_{r}$ by 1 column matrix. The Gaussian noise can also be represented as a $N_{r}$ by 1 column matrix. In [3] Section 3, the equations can be found to determine channel capacity. Note that the system model implicitly assumes a flat fading MIMO channel, i.e. channel coefficients are constant during the transmission of several symbols. Flat fading, or frequency nonselective fading, applies by definition to systems where the bandwidth of the transmitted signal is much smaller than the coherence bandwidth of the channel. All the frequency components of the transmitted signal undergo the same attenuation and phase shift propagation through the channel. The general equation for Shannon's capacity formula is:

$$
C=\log _{2}\left(1+y H^{2}\right)
$$

where $y$ is the received SNR and $H^{2}$ is the channel transfer characteristic.

For the cases of $N_{t}=3$ and 4 , codes with rates of $1 / 2$ and $3 / 4$ have full diversity of $3 N_{r}$ and $4 N_{r}$ respectively. For the case of $N_{t}=4$, to transmit at a rate of 1/2, 4 symbols are transmitted in 8-time intervals. The decision decoding matrix can be found in [4]. All other decoding metrics can easily be found in any Digital Communications book such as Digital Communications by John Proakis, 5th edition.

\subsection{Orthogonal Space Time Block Code Encoder}

The encoder consists of two parts: a constellation mapper and the space-time 
block encoder. All the modulation schemes mentioned have a specific mapper constellation it creates with the data it received. Figure 4 shows us the space time block encoder with the constellation mapper of QPSK and 16-QAM. The mapper takes the information source data and generates new sequences of blocks with each block made up of complex symbols $(M)$. All the symbols in a particular column of the transmission matrix are pulse shaped and modulated into a form suitable for simultaneous transmission over the channel by the transmit antennas [3]. The last part is the encoder. It will convert each block that the mapper has developed and create its own $M$ by $N_{t}$ matrix or temporal by spatial dimension aka the transmission matrix.

\section{Simulations}

Simulations were performed for STBC systems using multiple combinations of code rate, diversity, and antenna count. Each simulation is modulated using BPSK, QPSK, 16-QAM and 64-QAM. These modulation techniques provide excellent graphical data to show BER versus SNR for each STBC method.

A quasi-orthogonal space-time block code (QO-STBC) has a rate of 1 but suffers a loss of diversity gain, compared with an OSTBC such as Alamouti code. In this study [11], a code selection algorithm between QO-STBCs is proposed for diversity gain improvements of a system based on a QO-STBC with four transmit antennas. For the simulations that follow in Section 5, Alamouti and orthogonal cases are used. Each case has a channel model representing an nLOS environment with a Rayleigh fading channel used to mimic its characteristics. MATLAB code was written, compiled, and stored over a long period of time. The data log outputs from these files provided quick and easy graphs when plotted to visually organize and display the data. In the following performance analysis, we examine diversity with correlated Raleigh fading channels and, in special cases, correlated Rician fading channels. Research work performed outside of this paper extends the analysis focusing on the Nakagami fading model which is a distribution that provides greater flexibility in matching experimental data collected in a variety of fading environments.

\section{Performance Analysis}

From the graphs provided, several conclusions were based upon different factors. The selected factors include the number of transmit and receive antennas, diversity, code rate, and modulation technique. The decision to choose these factors were based on their individual impact to system performance while

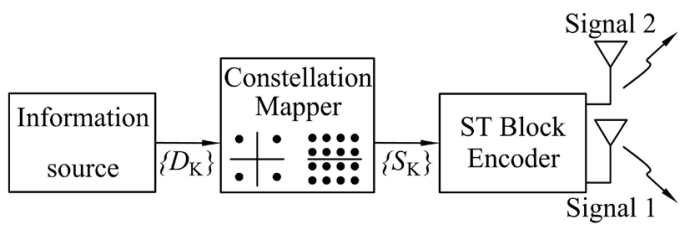

Figure 4. STB Encoder with constellation mapper. 
retaining simplicity to both simulate and graph. As you can see in Figure 5 for 1 receive antenna and transmit antennas ranging from 1 to 4 , performance is degraded when more bits per symbol are being transmitted. It is assumed that the total transmit power from the antennas in each scheme are the same as the total transmit power sent from a single transmit antenna. Modulating at low SNR and modulating at high SNR have different performance results. The callouts used to represent each case are $G_{x}$ and $H_{x}$ where $x$ denotes the number of transmit antennas and $\mathrm{G}$ being $1 / 2$ code rate and $\mathrm{H}$ being $3 / 4$ code rate. The lowest SNR was identified as G4 which is a code rate of 1/2 using 4 transmit antennas.

Conclusively, one can see how the purple and black lines overlap. This represents BPSK and QPSK modulation techniques. It is safe to say that the BER analysis of these two techniques is graphically the same. Do not forget that BPSK has the lowest data rate which is a major drawback. Low SNR in terms of any modulation technique goes from left-to-right as diamond, circle, star, square, triangle resulting in G4, G3, H4, H3, and G2. Though $\mathrm{H} 4$ has a lower SNR, G3 has a better overall BER performance. Considering high SNR, in terms of the 16-QAM and 64-QAM modulation techniques, the model displays diamond, star, circle, square, and triangle resulting in G4, H4, G3, H3, and H2.

Figure 6 is the same as Figure 5 except for using a fixed total of 4 receive antennas instead of 1 . For both low SNR and high SNR, regardless of modulation technique, the order of best performance from left-to-right is diamond, circle, star, square, and triangle resulting in G4, G3, H4, H3, and G2. This order matches Figure 5 when low SNR was used in conjunction with any modulation technique. A possible explanation for this could be $\mathrm{H} 4$ having a higher code rate at 3/4 when compared to G3 at 1/2. Lower channel gain per symbol is being produced creating a higher BER.

The last two cases varied the number of transmit antennas. For Figure 7, the number of receive antennas are varied with a fixed number of transmit antennas

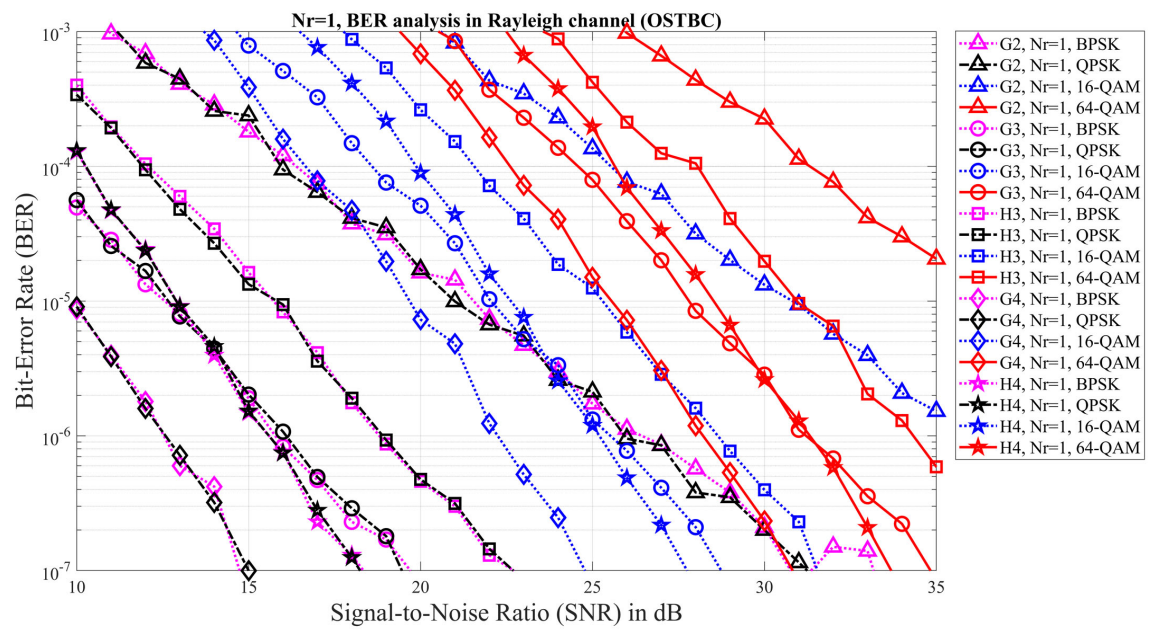

Figure 5. BER analysis of an OSTBC with 1 receive antenna and transmit antennas ranging from 1 to 4 using four modulation techniques. 


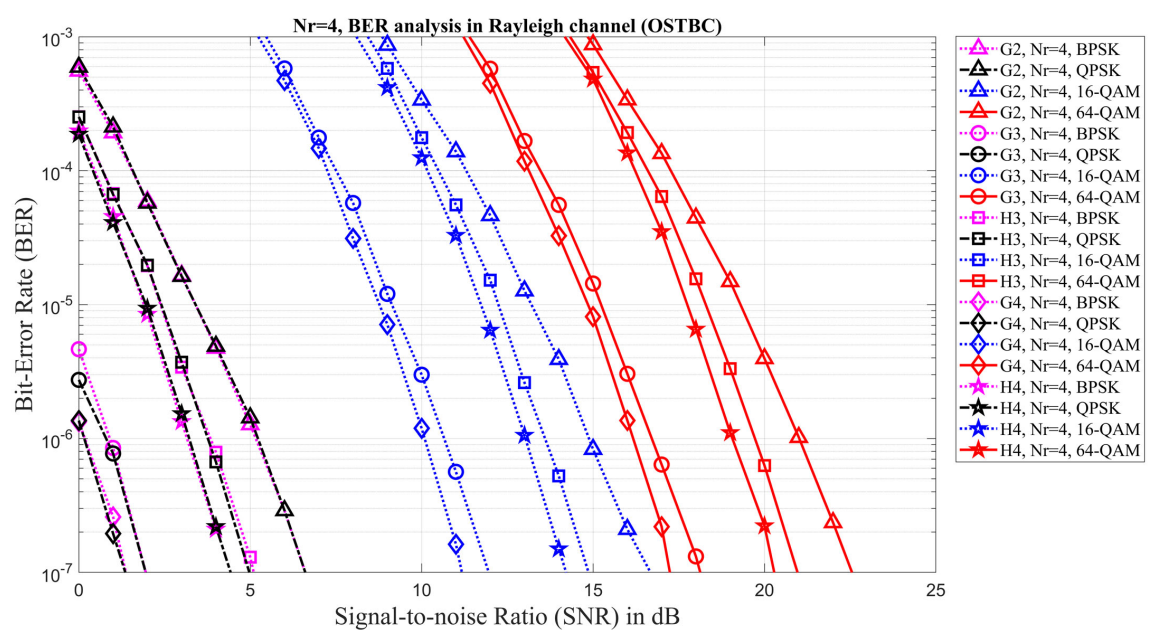

Figure 6. BER analysis of an OSTBC with 4 receive antennas and transmit antennas ranging from 1 to 4 using four modulation techniques.

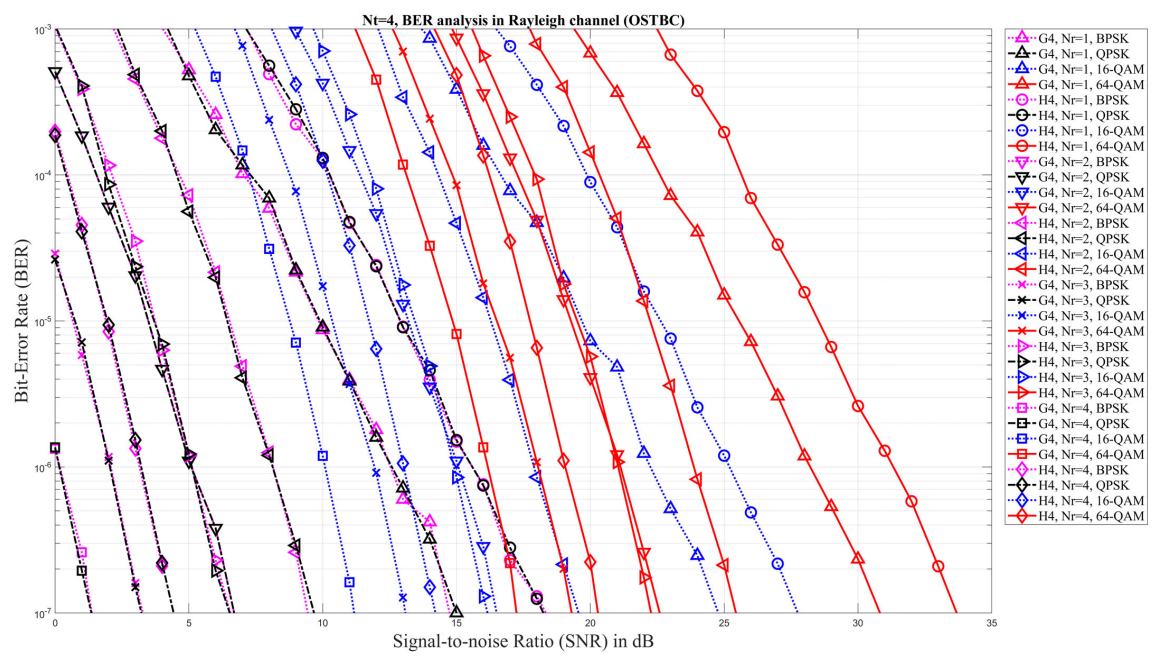

Figure 7. BER analysis of an OSTBC with 4 transmit antennas and receive antennas ranging from 1 to 4 using four modulation techniques.

resulting in an interesting pattern. If we pick a modulation type at random, say QPSK, and analyze its SNR as the number of receivers increase from 1 to 4 antennas, following the curves of each black line, we get an approximate $14 \mathrm{~dB}$ difference from $N_{r} 1$ to 4 using G4. The same is true with $\mathrm{H} 4$ determining that code rate has no influence on SNR. Analyzing the SNR in more depth shows the following: $N_{r}=1$ for G4 is exactly $15 \mathrm{~dB}$ and $N_{r}=2$ is approx. $7 \mathrm{~dB}$. This is a $8 \mathrm{~dB}$ differential when a receive antenna is added. $N_{r}$ from 2 to 3 is a $4 \mathrm{~dB}$ difference and $N_{r}$ from 3 to 4 is a $2 \mathrm{~dB}$ difference. Each additional receive antenna seems to decrease the SNR in half. Similarly, no significant difference between BPSK and QPSK is observed. A prominent difference that did present itself was that $G_{x}$ and $H_{x}$ varied in exactly $3 \mathrm{~dB}$ in every case with $G_{x}$ exhibiting a $3 \mathrm{~dB}$ gain over $H_{x}$ As the number of receive antennas increase, it is apparent the SNR decreases along with the BER. 
We can accurately see from Figure 8 with equal diversity across multiple modulation techniques that a lower number of transmit antennas along with a higher number of receive antennas achieved the best performance. Also, QPSK is the best modulation technique available from other choices such as 16-QAM and 64-QAM. One can assume that if fewer bits are being sent, this would decrease the BER. Through further examination, the squares and stars line up together representing G4 with $N_{r}=3$ and G2 with $N_{r}=6$. As stated above, a low number of transmit antennas used in conjunction with a high number of receive antennas show the best performance. So why does G4 with half the number of receive antennas using $N_{r}=3$ fit into that assumption? Evidently the use of higher transmit antennas with a low number of receive antenna overlap and cross in terms of their performance.

It has been observed that higher diversity gain is achieved with a greater number of antennas. This can be at either transmitter or receiver ends with a $3 \times$ 2 outperforming a $3 \times 1,2 \times 2$, and $2 \times 1$ antenna configuration. In contrast, more data rates are achieved in $2 \times 1$ and $2 \times 2$ when compared to $3 \times 1$ and $3 \times$ 2 cases with a different STBC matrix used in both cases. It has been observed that BPSK outperforms in terms of symbol error rate rather than QPSK, 16-QAM, and 64-QAM. BPSK is the simplest form of digital modulation. The main disadvantage with BPSK is low data rates as compared to QPSK which outputs almost the same performance [4]. In Figure 9, a simple flow chart of the previous MATLAB examples with QPSK modulation is shown. Some other analysis one can perform is in [3], where the calculation of the capacity and symbol error probability and bit-error probability are used. The capacity of MIMO systems using Rayleigh fading channels shows that multiple antennas increase capacity as well as increasing the number of receive antennas. This directly correlates with our performance analysis for the BER. QPSK seems to be the best performer overall but does not compete with the higher data-rate schemes like higher M-ary QAM schemes.

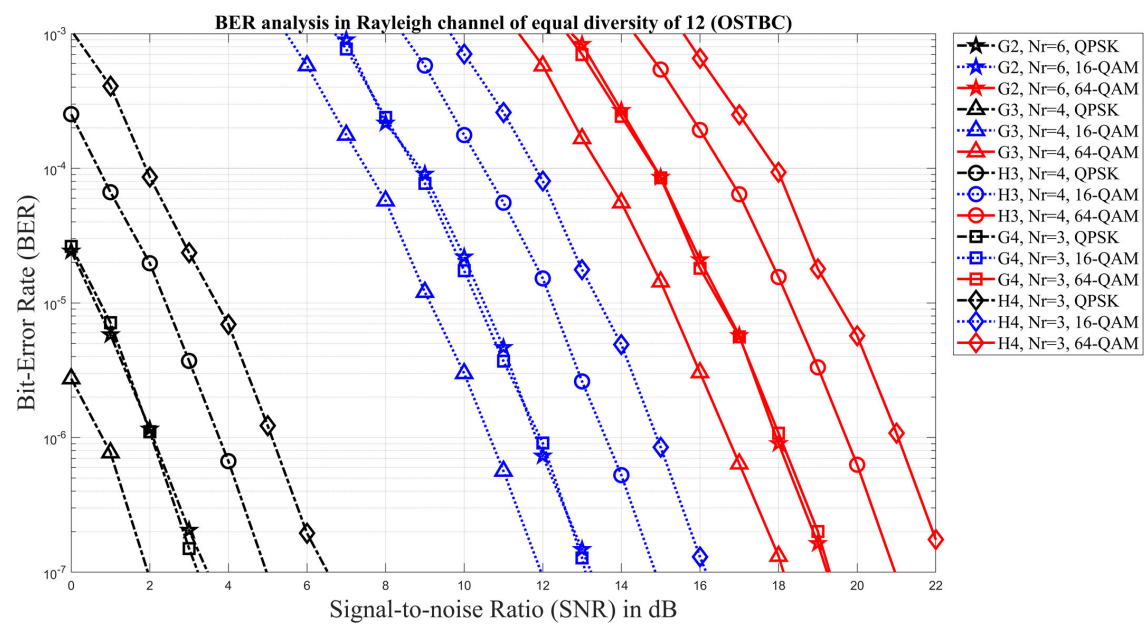

Figure 8. BER analysis of an OSTBC with equal gain using modulation techniques. 


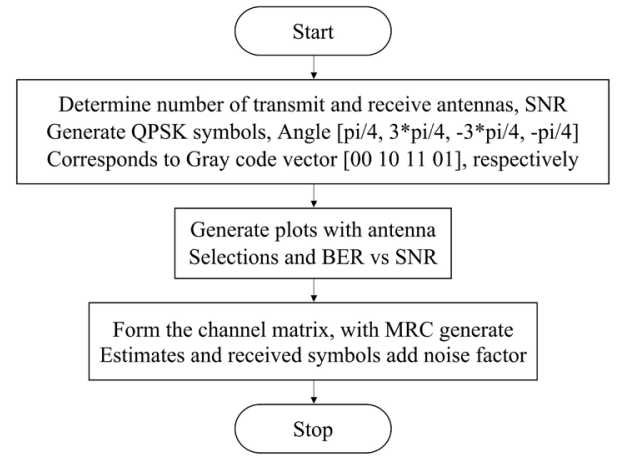

Figure 9. BER analysis flow chart of an OSTBC using QPSK modulation.

\section{Conclusions}

This paper provides the fundamentals of MIMO systems as we looked in depth at STBC schemes and how they perform in Rayleigh fading channels for different modulation techniques. Wireless systems and their associated technologies are in high demand. MIMO systems are widely used for the ease of planning and a limited number of installed antennas. The amount of time and money that go into these systems, especially with the use of STBC, allows it to be further studied, researched, and enhanced by designers and engineers. Performance of a MIMO system using STBC is heavily impacted on the number of antennas being used for transmissions. Modulation techniques such as BPSK and QPSK gave an overall lower bit-error rate. The graphical data plotted for a combination of systems concluded that two transmit antennas achieved full rate and full diversity. It was when the number of transmit antennas increased beyond two that a decrease in performance data was observed. In order to significantly increase performance output, the number of receivers were increased. This had the largest impact on the system in terms of a higher SNR in conjunction with a lower BER.

One might not want to have many receivers due to its impractical deployment in real-world installations. As a tradeoff, the simulations presented in Section 5 showed that in some cases where a specific modulation scheme was being used, that a higher number of transmit antennas produced the same performance levels as compared to increasing the receive antennas. This layout is ideal for base stations utilizing a limited amount of mobile stations especially for cellular data. Only requiring extra antennas at the base station is much more economical for cost and physical deployment rather than having multiple receivers. For large MIMO installations such as those serving cellular 5G, huge quantities of antennas will allow cell networks to provide clear, reliable communications. With limited frequency spectrum available and a fast-growing number of subscribers and end users, MIMO systems will lead the way in the wireless industry as channel correlation, interference reduction and modulation techniques are explored and improved upon.

\section{Conflicts of Interest}

The author declares no conflicts of interest regarding the publication of this paper. 


\section{References}

[1] Salehi Nobandegani, K. and Azmi, P. (2009) A New Low SNR, Correlated Fading-Suited Space-Time Block Code Based on Zero-Padding and Unitary Transforms. Telecommunication Systems, 41, 243-253. https://doi.org/10.1007/s11235-009-9159-8

[2] Alamouti, S.M. (1995) A Simple Transmit Diversity Technique for Wireless Communications. IEEE Journal on Selected Areas in Communications, 16, 1451-1458. https://doi.org/10.1109/49.730453

[3] Veeranna, D. and Nidhi, R. (2012) Performance Analysis on Different Modulation Techniques of MIMO in Multipath Fading Channel. IOSR Journal of Engineering, 2, 738-743. https://doi.org/10.9790/3021-0204738743

[4] Amin, M.R. and Trapasiya, S.D. (2012) Performance Optimization of MIMO Using Space-Time Block Coding Scheme. Procedia Engineering, 38, 3518-3527. https://doi.org/10.1016/j.proeng.2012.06.406

[5] Ham, J., Kim, K., Shin, M. and Lee, C. (2010) Performance Analysis of Code Selection Algorithm Based on Quasi-Orthogonal Space-Time Block Code. IET Communications, 4, 1847-1854. https://doi.org/10.1049/iet-com.2010.0055

[6] Sin, L.C.H. (2003) Semi-Blind Interference Suppression Algorithm for a Downlink MC-CDMA System That Employs Space Time Block Code. ProQuest Dissertations \& Theses Global: The Sciences and Engineering Collection, Order No. MQ84290, University of Toronto (Canada), Ann Arbor.

[7] Basar, E. and Aygolu, U. (2010) High-Rate Full-Diversity Space-Time Block Codes for Three and Four Transmit Antennas. IET Communications, 3, 1371-1378. https://doi.org/10.1049/iet-com.2008.0697

[8] Sujatha, B.R. (2012) Space-Time Block Coding (STBC) for Wireless Networks. International Journal of Distributed and Parallel Systems (IJDPS), 3, 183-195. https://doi.org/10.5121/ijdps.2012.3419

[9] Tarokh, V., Seshadri, N. and Calderbank A.R. (1998) Space-Time Codes for High Data Rate Wireless Communication: Performance Analysis and Code Construction. IEEE Transactions on Information Theory, 44, 744-765. https://doi.org/10.1109/18.661517

[10] Riediger, M. and Ho, P. (2005) Non-Coherent Detection of Alamouti Space-Time Block Code. IEEE Global Telecommunications Conference, 6, 2956-2961.

[11] Bardhan, S. (2012) Capacity and Performance Analysis of MIMO-STBC in Rayleigh Fading Channels. International Journal of Engineering Research \& Technology (IJERT), 1.

https://www.ijert.org/capacity-and-performance-analysis-of-mimo-stbc-in-rayleigh -fading-channels 